\title{
QUADRATIC DIFFERENTIAL EQUATIONS IN THE COMPLEX DOMAIN I
}

\author{
NORA C. HOPKINS
}

\begin{abstract}
By complexifying all of the variables of an ordinary real quadratic vector differential equation to get a differential equation over $\mathbb{C}$, it is shown that the solution to the complex differential equation can be uniquely defined on an open star-shaped subset of $\mathbb{C}$, dependent on the initial point, containing the maximum interval of existence of the real differential equation. Complex conjugation is shown to commute with solving the differential equation on this complex domain, and well-known algebraic properties of the solutions to the real differential equation are generalized to the equation over $\mathbb{C}$.
\end{abstract}

\section{INTRODUCTION}

There is a nonassociative algebra structure on $\mathbb{R}^{n}$ associated in a natural way with each quadratic differential equation on $\mathbb{R}^{n}$. In $[3]$ the author and M. Kinyon showed that the automorphisms of the nonassociative algebra structure and their eigenspaces play a role in the qualitative properties of the differential equation, but that investigation was limited to real eigenvalues. The present paper and its sequel [2] address the natural question of whether the nonreal eigenvalues of an automorphism and the associated eigenspaces are useful in understanding the real differential equation. Of course, this will necessitate treating all of the variables, including the independent variable time, as complex, since a trajectory with real initial point will not intersect such an eigenspace at a real time.

Throughout, all vector spaces and algebras will be finite dimensional over the field $K$, where $K$ is either $\mathbb{R}$ or $\mathbb{C}$.

A quadratic differential equation on $K^{n}$ is an autonomous system of ordinary differential equations of the form

$$
\begin{aligned}
\frac{d z_{1}}{d t} & =q_{1}\left(z_{1}, \ldots, z_{n}\right) \\
\vdots & \\
\frac{d z_{n}}{d t} & =q_{n}\left(z_{1}, \ldots, z_{n}\right),
\end{aligned}
$$

where each $q_{i}: K^{n} \rightarrow K$ is a homogeneous quadratic polynomial in the variables $z_{i}, i=1, \ldots, n$. We will assume throughout that the coefficients occurring in the $q_{i}$ 's are real. With this assumption we actually have three systems coming from (1.1): one in which we treat all variables as real, one in which we treat all variables as complex, and one in which $t$ is real and the $z_{i}$ 's are allowed to be complex. All three systems will be important for our work here.

Received by the editors March 11, 2009.

2010 Mathematics Subject Classification. Primary 34M99, 34C14, 17A36. 
There is a standard homogenization trick for embedding systems of the form (1.1) for which the $q_{i}$ are quadratic but not homogeneous into quadratic differential equations on $K^{n+1}$ ([6] or [12]). Thus, the Lorenz model of thermal convection, linear control systems with quadratic cost function, the Euler equations for the motion of rotating rigid bodies in the absence of external forces, and Lotka-Volterra predator-prey models are all quadratic differential equations after homogenization.

We will treat (1.1) as the following vector differential equation over $K$ :

$$
\frac{d Z}{d t}=Z^{2}
$$

where

$$
Z=\left(\begin{array}{c}
z_{1} \\
\vdots \\
z_{n}
\end{array}\right) \text { and } Z^{2}:=\left(\begin{array}{c}
q_{1}\left(z_{1}, \ldots, z_{n}\right) \\
\vdots \\
q_{n}\left(z_{1}, \ldots, z_{n}\right)
\end{array}\right)
$$

If the initial condition for $(1.1)$ is $z_{i}(0)=p_{i}, i=1, \ldots, n$, then the initial condition for $(1.2)$ is $Z(0)=P$, where $P=\left(\begin{array}{c}p_{1} \\ \vdots \\ p_{n}\end{array}\right)$. We will assume hereafter that $P \neq 0$.

Throughout, $Z_{K}(t, P)$ will denote the solution to (1.2) over $K$ with $Z_{K}(0, P)=P$. The maximum interval of existence of $Z_{\mathbb{R}}(t, P)$ will be denoted by $(\alpha(P), \omega(P))$ or $(\alpha, \omega)$ if $P$ is understood. The first issue we must deal with in $\S 2$ is to find a domain for $Z_{\mathbb{C}}(t, P)$ containing $(\alpha(P), \omega(P))$ and on which $Z_{\mathbb{C}}(t, P)$ is unique. Then we will be able to see (Corollary 2.6) that $Z_{\mathbb{C}}(t, P)=Z_{\mathbb{R}}(t, P)$ for $t \in(\alpha(P), \omega(P))$ and thereafter simply refer to $Z(t, P)$.

It is possible to define a "product" on $K^{n}$ from the definition of $Z^{2}$ by

$$
Z W:=\frac{1}{2}\left\{(Z+W)^{2}-Z^{2}-W^{2}\right\}
$$

for $Z, W \in K^{n}$. This product is not necessarily associative, so $K^{n}$ with this product is an example of a nonassociative algebra as defined below. First, we give an example:

Example 1.4. For the differential equation

$$
\begin{aligned}
& \frac{d z_{1}}{d t}=z_{2}^{2} \\
& \frac{d z_{2}}{d t}=z_{1}^{2}
\end{aligned}
$$

$$
\begin{gathered}
Z^{2}=\left(\begin{array}{c}
z_{2}^{2} \\
z_{1}^{2}
\end{array}\right) \text { if } Z=\left(\begin{array}{c}
z_{1} \\
z_{2}
\end{array}\right) \text {. Thus if } Z=\left(\begin{array}{c}
z_{1} \\
z_{2}
\end{array}\right) \text { and } W=\left(\begin{array}{l}
w_{1} \\
w_{2}
\end{array}\right),(1.3) \text { gives } \\
Z W=\frac{1}{2}\left\{\left(\begin{array}{c}
\left(z_{2}+w_{2}\right)^{2} \\
\left(z_{1}+w_{1}\right)^{2}
\end{array}\right)-\left(\begin{array}{c}
z_{2}^{2} \\
z_{1}^{2}
\end{array}\right)-\left(\begin{array}{c}
w_{2}^{2} \\
w_{1}^{2}
\end{array}\right)\right\}=\left(\begin{array}{l}
z_{2} w_{2} \\
z_{1} w_{1}
\end{array}\right) ;
\end{gathered}
$$

i.e., the product on $K^{2}$ coming from (1.4.1) is

$$
\left(\begin{array}{l}
z_{1} \\
z_{2}
\end{array}\right)\left(\begin{array}{l}
w_{1} \\
w_{2}
\end{array}\right)=\left(\begin{array}{l}
z_{2} w_{2} \\
z_{1} w_{1}
\end{array}\right)
$$

Note that

$$
\left(\left(\begin{array}{l}
z_{1} \\
z_{2}
\end{array}\right)\left(\begin{array}{l}
w_{1} \\
w_{2}
\end{array}\right)\right)\left(\begin{array}{l}
a_{1} \\
a_{2}
\end{array}\right)=\left(\begin{array}{l}
z_{1} w_{1} a_{2} \\
z_{2} w_{2} a_{1}
\end{array}\right) \text { and }\left(\begin{array}{l}
z_{1} \\
z_{2}
\end{array}\right)\left(\left(\begin{array}{l}
w_{1} \\
w_{2}
\end{array}\right)\left(\begin{array}{l}
a_{1} \\
a_{2}
\end{array}\right)\right)=\left(\begin{array}{l}
z_{2} w_{1} a_{1} \\
z_{1} w_{2} a_{2}
\end{array}\right),
$$

so this product does not satisfy the associative law. 
A nonassociative algebra over $K$ is a vector space $\mathfrak{A}$ over $K$ with a bilinear mapping $\mathfrak{A} \times \mathfrak{A} \rightarrow \mathfrak{A}$ called the product or multiplication and denoted by $(X, Y) \mapsto$ $X Y$ (or sometimes $X \cdot Y$ ). $\mathfrak{A}$ is commutative if $X Y=Y X$ for all $X, Y \in \mathfrak{A}$. Note that if $\mathfrak{A}_{\mathbb{R}}$ is an algebra over $\mathbb{R}$, then $\mathfrak{A}_{\mathbb{C}}:=\mathfrak{A}_{\mathbb{R}} \otimes_{\mathbb{R}} \mathbb{C}=\mathfrak{A}_{\mathbb{R}} \oplus i \mathfrak{A}_{\mathbb{R}}$ with the product defined by

$$
(X+i Y)(Z+i W):=(X Z-Y W)+i(X W+Y Z)
$$

for $X, Y, Z, W \in \mathfrak{A}_{\mathbb{R}}$ is an algebra over $\mathbb{C}$ and $\mathfrak{A}_{\mathbb{C}}$ is commutative if $\mathfrak{A}_{\mathbb{R}}$ is commutative. Of course, $\mathfrak{A}_{\mathbb{C}}$ with the product defined by (1.5) is also an algebra over $\mathbb{R}$, and when we want to think of it as such, it will be denoted by $\mathbb{R}_{\mathbb{C}}$.

Hereafter, $\mathfrak{A}_{K}$ will be the nonassociative algebra $K^{n}$ with product defined by (1.3), where $Z^{2}$ is defined from (1.2). Note that $\mathfrak{A}_{K}$ is commutative and that the product on $\mathfrak{A}_{\mathbb{C}}$ is also given by (1.5). Thus, when treating all of the variables in (1.1) as real, we will be working in $\mathfrak{A}_{\mathbb{R}}$, and when treating all variables as complex we will be working in $\mathfrak{A}_{\mathbb{C}}$. When treating $t$ as real and all other variables as complex in (1.1), we will be working in $\mathbb{R}^{\mathfrak{A}_{\mathbb{C}}}$.

Returning to the general theory of nonassociative algebras, if $\mathfrak{A}$ is a nonassociative algebra over $K$, a $K$-subspace $\mathcal{B}$ of $\mathfrak{A}$ is a subalgebra of $\mathfrak{A}$ if $\mathcal{B}$ is closed under the algebra product of $\mathfrak{A}$. Given $S \subseteq \mathfrak{A}$, the subalgebra generated by $S$, denoted $\mathfrak{A}(S)$ is the unique smallest subalgebra of $\mathfrak{A}$ containing $S$. For $P \in \mathfrak{A}, \mathfrak{A}(\{P\})$ will be denoted by $\mathfrak{A}(P)$. Since we will be changing fields at times, we will indicate this with a subscript; that is, $\mathfrak{A}_{\mathbb{R}}(S)$ will be the unique smallest subalgebra of $\mathbb{R}_{\mathbb{C}}$ containing $S$ and $\mathfrak{A}_{\mathbb{C}}(S)$ will be the unique smallest subalgebra of $\mathfrak{A}_{\mathbb{C}}$ containing $S$. Obviously, $\mathfrak{A}_{\mathbb{R}}(S) \subseteq \mathfrak{A}_{\mathbb{C}}(S)$, and if $S \subseteq \mathfrak{A}_{\mathbb{R}}$, then $\mathfrak{A}_{\mathbb{R}}(S)$ is the unique smallest subalgebra of $\mathfrak{A}_{\mathbb{R}}$ containing $S$ since $\mathfrak{A}_{\mathbb{R}}$ is a subalgebra of $\mathbb{R}_{\mathbb{C}}$.

Given $K$-subspaces $\mathcal{B}$ and $\mathcal{C}$ of a nonassociative algebra $\mathfrak{A}$ over $K, \mathcal{B C}$ is the $K$ subspace of all finite sums of products of elements of $\mathcal{B}$ with elements $\mathcal{C}$, viz $\mathcal{B C}:=$ $\left\{\sum Z_{i} W_{i} \mid Z_{i} \in \mathcal{B}, W_{i} \in \mathcal{C}\right\}$. Assuming $\mathfrak{A}$ is commutative, a subalgebra $\mathcal{B}$ is nilpotent if $\mathcal{B}^{n}=0$ for some $n \in \mathbb{N}$, where $\mathcal{B}^{1}:=\mathcal{B}$ and $\mathcal{B}^{j+1}:=\mathcal{B}^{j} \mathcal{B}$.

A number of authors $([5,6], 7], 8,9],[1])$ have shown that the algebraic properties of $\mathfrak{A}_{\mathbb{R}}$ give information about the solutions of (1.2) as a real differential equation. A basic result is the following.

Theorem 1.6. (i) [9]: If $\mathcal{B}_{\mathbb{R}}$ is a subalgebra of $\mathfrak{A}_{\mathbb{R}}$ and $P \in \mathcal{B}_{\mathbb{R}}$, then $Z_{\mathbb{R}}(t, P) \in \mathcal{B}_{\mathbb{R}}$ for all $t \in(\alpha(P), \omega(P))$. In particular, $Z_{\mathbb{R}}(t, P) \in \mathfrak{A}_{\mathbb{R}}(P)$ for all $t \in(\alpha(P), \omega(P))$.

(ii) [5], 11]: If $\mathcal{B}_{\mathbb{R}}$ is a nilpotent subalgebra of $\mathfrak{A}_{\mathbb{R}}$ and $P \in \mathcal{B}_{\mathbb{R}}$, the $Z_{\mathbb{R}}(t)$ is a polynomial in $t$ (and in $P$ ) and $(\alpha(P), \omega(P))=\mathbb{R}$.

This result will generalize immediately to $Z_{\mathbb{C}}(t, P)$ once the domain of $\mathbb{Z}_{\mathbb{C}}(t, P)$ is defined; see Theorem 2.4. Also, by Theorem 1.6(i) since $\mathfrak{A}_{\mathbb{R}}$ is a subalgebra of $\mathbb{R} \mathfrak{A}_{\mathbb{C}}$, if $P \in \mathfrak{A}_{\mathbb{R}}$, then $Z_{\mathbb{R}}(t, P)$ is unaffected by thinking of $P$ as an element of $\mathbb{R}_{\mathbb{C}}$.

An automorphism of a nonassociative algebra $\mathfrak{A}$ over $K$ is an invertible $K$-linear transformation $A: \mathfrak{A} \rightarrow \mathfrak{A}$ such that $A(X Y)=A(X) \cdot A(Y)$ for all $X, Y \in \mathfrak{A}$. $\operatorname{Aut}(\mathfrak{A})$ will denote the group of automorphisms of $\mathfrak{A}$. A derivation of $\mathfrak{A}$ is a $K$ linear transformation $D: \mathfrak{A} \rightarrow \mathfrak{A}$ such that $D(X Y)=D(X) \cdot Y+X \cdot D(Y)$ for all $X, Y \in \mathfrak{A}$. Der $(\mathfrak{A})$ will denote the Lie algebra of all derivations of $\mathfrak{A}$. As noted in [3] an automorphism of $\mathfrak{A}_{K}$ is a symmetry of (1.2) in the sense of Lie and a derivation of $\mathfrak{A}_{K}$ is an infinitesmal symmetry of (1.2). Recall [7] that if $D \in \operatorname{Der}\left(\mathfrak{A}_{K}\right)$, then $\exp D \in \operatorname{Aut}\left(\mathfrak{A}_{K}\right)$, where $\exp D:=\sum_{n=0}^{\infty} \frac{1}{n !} D^{n}$. The relationships among $\operatorname{Aut}\left(\mathfrak{A}_{\mathbb{R}}\right)$, 
$\operatorname{Aut}\left(\mathfrak{A}_{\mathbb{C}}\right)$, Aut $\left(\mathbb{R}_{\mathbb{A}} \mathfrak{A}_{\mathbb{C}}\right)$ and among Der $\left(\mathfrak{A}_{\mathbb{R}}\right)$, Der $\left(\mathfrak{A}_{\mathbb{C}}\right)$ and $\operatorname{Der}\left(\mathbb{R}_{\mathbb{C}}\right)$ are given by the following proposition.

Proposition 1.7. (i) Suppose $A \in A u t\left(\mathfrak{A}_{\mathbb{R}}\right)$. Extend $A$ to $\mathfrak{A}_{\mathbb{C}}$ by

$$
A(X+i Y):=A(X)+i A(Y)
$$

for $X, Y \in \mathfrak{A}_{\mathbb{R}}$. Then $A \in A$ ut $\left(\mathfrak{A}_{\mathbb{C}}\right)$. This extension defines a group monomorphism from $\operatorname{Aut}\left(\mathfrak{A}_{\mathbb{R}}\right)$ to $\operatorname{Aut}\left(\mathfrak{A}_{\mathbb{C}}\right)$.

(ii) $\operatorname{Aut}\left(\mathfrak{A}_{\mathbb{C}}\right) \subseteq \operatorname{Aut}\left(\mathbb{R}_{\mathbb{A}}\right)$ and $\operatorname{Der}\left(\mathfrak{A}_{\mathbb{C}}\right) \subseteq \operatorname{Der}\left(\mathbb{R}_{\mathbb{A}}\right)$.

(iii) Define complex conjugation on $\mathbb{R}_{\mathbb{C}}$ by

$$
\overline{X+i Y}:=X-i Y
$$

for $X, Y \in \mathfrak{A}_{\mathbb{R}}$. Then complex conjugation is an automorphism of $\mathbb{R}_{\mathbb{C}}$.

(iv) Suppose $A \in A$ ut $\left(\mathfrak{A}_{\mathbb{C}}\right)$. Define $\bar{A}: \mathfrak{A}_{\mathbb{C}} \rightarrow \mathfrak{A}_{\mathbb{C}}$ by $\bar{A}(P)=\overline{A(\bar{P})}$. Then $\bar{A} \in \operatorname{Aut}\left(\mathfrak{A}_{\mathbb{C}}\right)$. Moreover $A$ is an extension of an automorphism of $\mathfrak{A}_{\mathbb{R}}$ as defined in (i) iff $\bar{A}=A$.

(v) Suppose $D \in \operatorname{Der}\left(\mathfrak{A}_{\mathbb{R}}\right)$. Extend $D$ to $\mathfrak{A}_{\mathbb{C}}$ by

$$
D(X+i Y)=D(X)+i D(Y)
$$

for $X, Y \in \mathfrak{A}_{\mathbb{R}}$. Then $D \in \operatorname{Der}\left(\mathfrak{A}_{\mathbb{C}}\right)$.

(vi) Suppose $D \in \operatorname{Der}\left(\mathfrak{A}_{\mathbb{C}}\right)$. Define $\bar{D}: \mathfrak{A}_{\mathbb{C}} \rightarrow \mathfrak{A}_{\mathbb{C}}$ by $\bar{D}(P)=\overline{D(\bar{P})}$. Then $\bar{D} \in \operatorname{Der}\left(\mathfrak{A}_{\mathbb{C}}\right)$. Moreover $D$ is an extension of a derivation of $\mathfrak{A}_{\mathbb{R}}$ as defined in $(v)$ iff $\bar{D}=D$.

If $\mathfrak{A}$ is a nonassociative algebra over $K$ and $B: \mathfrak{A} \rightarrow \mathfrak{A}$ is $K$-linear, then for $b \in K, E_{b}(B):=\{X \in \mathfrak{A} \mid B(X)=b X\}$. We will write $A Z_{K}(t, P)$ for $A\left(Z_{K}(t, P)\right)$ hereafter. One result to be generalized to complex quadratic differential equations (Theorems 3.1 and 3.5) is the following.

Theorem $1.8([5],[2])$. (i) If $A \in \operatorname{Aut}\left(\mathbb{R}_{\mathbb{R}} \mathfrak{A}_{\mathbb{C}}\right)$ and $P \in E_{1}(A)$, then $Z_{\mathbb{R}}(t, P) \in$ $E_{1}(A)$ for all $t \in(\alpha(P), \omega(P))$.

(ii) Suppose $s \in \mathbb{R}-\{0\}$. Then $(\alpha(s P), \omega(s P))=s^{-1} \cdot(\alpha(P), \omega(P))$ and

$$
Z_{\mathbb{R}}(t, s P)=s Z_{\mathbb{R}}(s t, P)
$$

for all $t \in(\alpha(s P), \omega(s P))$.

(iii) Suppose $A \in A$ ut $\left(\mathbb{R}_{\mathbb{A}} \mathbb{C}_{\mathbb{C}}\right)$. Then $(\alpha(P), \omega(P))=(\alpha(A P), \omega(A P))$ and

$$
A Z_{\mathbb{R}}(t, P)=Z_{\mathbb{R}}(t, A P)
$$

for all $t \in(\alpha(P), \omega(P))$.

(iv) Suppose $A \in A u t\left(\mathbb{R}_{\mathbb{C}}\right)$ and $P \in E_{a}(A)$. Then $(\alpha(P), \omega(P))=a^{-1} \cdot(\alpha(P), \omega(P))$ and

$$
A Z_{\mathbb{R}}(t, P)=a Z_{\mathbb{R}}(a t, P)
$$

for all $t \in(\alpha(P), \omega(P))$. If a is not a root of 1 , then $(\alpha(P), \omega(P))=\mathbb{R}$ and $Z_{\mathbb{R}}(t, P)$ is a polynomial in $t$ (and $P$ ).

(v) Suppose $D \in \operatorname{Der}\left(\mathbb{R}_{\mathbb{C}}\right)$ and $P \in E_{a}(D)$ for $a \neq 0$. Then $Z_{\mathbb{R}}(t, P)$ is a polynomial in $t$ (and $P$ ) and $(\alpha(P), \omega(P))=\mathbb{R}$.

Here for $S \subseteq \mathbb{C}$ and $a \in \mathbb{C}, a \cdot S:=\{a s \mid s \in S\}$.

The following is an immediate corollary to Theorem 1.7(iii) and Theorem 1.8(iii). 
Corollary 1.9. $(\alpha(P), \omega(P))=(\alpha(\bar{P}), \omega(\bar{P}))$ and for all $t \in(\alpha(P), \omega(P))$,

$$
\overline{Z_{\mathbb{R}}(t, P)}=Z_{\mathbb{R}}(t, \bar{P}) .
$$

It is important to note that some of the results of [3] do not generalize to the complex case, e.g. Corollary 3.6 of [3]; see Example 4.5 below.

In $\S 2$ we define the domain $S_{P}$ of $Z_{\mathbb{C}}(t, P)$ (Theorem 2.4) and show its relationship to $(\alpha(P), \omega(P))$ (Theorem 2.5). We also show $Z_{\mathbb{C}}(t, P)=Z_{\mathbb{R}}(t, P)$ for $t \in(\alpha, \omega)$ (Corollary 2.6). In $\S 3$ we generalize Theorem 1.8 to $Z(t, P)$ on $S_{P}$ (Theorems 3.1 and 3.5) and show the relationship between $S_{P}$ and $\left(\alpha\left(e^{i \theta} P\right), \omega\left(e^{i \theta} P\right)\right)$ for $\theta \in[0,2 \pi]$ (Corollaries 3.2 and 3.4). In $\S 4$ we show $\overline{S_{P}}=S_{\bar{P}}$ and $\overline{Z(t, P)}=Z(\bar{t}, \bar{P})$ for $t \in S_{P}$ (Theorem 4.1). $\S 5$ considers the relationship between $S_{P}$ and $S_{Q}$, where $Q=Z(s, P)$ for some $s \in S_{P}$. In $\S 6$ we give some results guaranteeing $Z(t, P)$ is periodic. Of particular note is Theorem 6.4, which says that if $P \in \mathfrak{A}_{\mathbb{R}}$ and $A \in \operatorname{Aut}\left(\mathfrak{A}_{\mathbb{R}}\right)$ with $A Z\left(t_{0}, P\right) \in E_{-1}(A)$ for some $t_{0}$ not real, then $Z(t, P)$ is periodic provided $S_{P}=\mathbb{C}$. We note that the approach used here to determine the domain of existence $S_{p}$ is not the usual one found in [1].

\section{Definition of $S_{P}$}

Recall that $S \subseteq \mathbb{C}$ is star-shaped with respect to $s$ if $a \in S$ implies $s+t(a-s) \in S$ for all $t \in[0,1]$; i.e., the line segment in the complex plane from $a$ to $s$ is contained in $S$. The following proposition has the properties of the star-shaped regions we will need.

Proposition 2.1. Suppose $S, S_{1}, S_{2}$, and $S_{j}$ for $j \in J$ are open subsets of $\mathbb{C}$ which are all star-shaped with respect to 0 . Then we have the following:

(i) $S$ is connected and simply connected.

(ii) If $s \in \mathbb{C}$ with $s \neq 0$, then $s \cdot S$ is star-shaped with respect to 0 and $S+s:=$ $\{x+s \mid x \in S\}$ is star-shaped with respect to $s$.

(iii) $\bigcup_{j \in J} S_{j}$ is star-shaped with respect to 0 .

(iv) $S_{1} \cap S_{2}$ is star-shaped with respect to 0 .

Lemma 2.2. Suppose $P \in \mathfrak{A}_{\mathbb{C}}$ and $S$ is an open subset of $\mathbb{C}$ which is star-shaped with respect to 0 and on which $Z_{\mathbb{C}}(t, P)$ can be defined. Then $Z_{\mathbb{C}}(t, P)$ is defined uniquely on $S$.

Proof. Suppose $\theta \in[0,2 \pi]$. There is an $R>0$ such that the Maclauren series for both $Z_{\mathbb{C}}(t, P)$ and $Z_{\mathbb{C}}\left(t, e^{i \theta} P\right)$ converge for $|t|<R$ and such that $t \in S$ for $|t|<R$. Then $Z_{\mathbb{C}}\left(t, e^{i \theta} P\right)=e^{i \theta} Z_{\mathbb{C}}\left(e^{i \theta} t, P\right)$ for $|t|<R$ since both sides are the unique analytic solution to $\frac{d Z}{d t}=Z^{2}$ and $Z(0)=e^{i \theta} P$. In particular, for $s \in(-R, R)$ we have $Z_{\mathbb{C}}\left(s, e^{i \theta} P\right)=e^{i \theta} Z_{\mathbb{C}}\left(e^{i \theta} s, P\right)$. Also for $s \in(-R, R), Z_{\mathbb{C}}\left(s, e^{i \theta} P\right)=Z_{\mathbb{R}}\left(s, e^{i \theta} P\right)$ by uniqueness. Now if $S \cap e^{i \theta} \mathbb{R}=e^{i \theta} \cdot\left(\beta_{\theta}, \gamma_{\theta}\right)$, then $0 \in\left(\beta_{\theta}, \gamma_{\theta}\right)$, so $Z_{\mathbb{C}}\left(e^{i \theta} s, P\right)=$ $e^{i \theta} Z_{\mathbb{R}}\left(s, e^{i \theta} P\right)$ for $s \in\left(\beta_{\theta}, \gamma_{\theta}\right)$ by the uniqueness of $Z_{\mathbb{R}}\left(s, e^{i \theta} P\right)$ computed using $\mathbb{R} \mathfrak{A}_{\mathbb{C}}$. Hence $Z_{\mathbb{C}}(t, P)$ is uniquely defined on $S$.

Corollary 2.3. Suppose $P \in \mathfrak{A}_{\mathbb{C}}$ and $S$ is an open subset of $\mathbb{C}$ which is starshaped with respect to 0 and on which $Z_{\mathbb{C}}(t, P)$ can be defined. Then $S \cap e^{i \theta} \mathbb{R} \subseteq$ $\left(\alpha\left(e^{i \theta} P\right), \omega\left(e^{i \theta} P\right)\right)$ for $\theta \in[0,2 \pi]$.

Now we can define the domain $S_{P}$ of $Z_{\mathbb{C}}(t, P)$ that we will use hereafter. 
Theorem 2.4. Let $\mathcal{S}$ be the collection of open subsets of $\mathbb{C}$ which are star-shaped with respect to 0 and on which $Z_{\mathbb{C}}(t, P)$ can be defined. Let $S_{P}=\bigcup_{S \in \mathcal{S}} S$. Then $Z_{\mathbb{C}}(t, P)$ is uniquely defined on $S_{P}$.

Proof. If $S_{1}, S_{2} \in \mathcal{S}$, then $S_{1} \cap S_{2} \in \mathcal{S}$ by Proposition 2.1(iv), and hence if $Z_{\mathbb{C}}(t, P, S)$ is $Z_{\mathbb{C}}(t, P)$ as defined on $S \in S$, then $\left.Z_{\mathbb{C}}\left(t, P, S_{1}\right)\right|_{S_{1} \cap S_{2}}=\left.Z_{\mathbb{C}}\left(t, P, S_{2}\right)\right|_{S_{1} \cap S_{2}}$ by Lemma 2.2. Hence we define $Z_{\mathbb{C}}(t, P)$ on $S_{P}$ by $Z_{\mathbb{C}}(t, P):=Z_{\mathbb{C}}(t, P, S)$ for $t \in S$ and $S \in \mathcal{S}$.

Clearly, $S_{P}$ is the largest open subset of $\mathbb{C}$ which is star-shaped with respect to 0 and on which $Z_{\mathbb{C}}(t, P)$ can be defined.

The advantage to using $S_{P}$ as the domain of $Z_{\mathbb{C}}(t, P)$ can be seen from the following two results.

Theorem 2.5. $S_{P} \cap \mathbb{R}=(\alpha(P), \omega(P))$.

Proof. By Corollary 2.3, $S_{P} \cap \mathbb{R} \subseteq(\alpha(P), \omega(P))$. Now suppose $[\beta, \gamma] \subseteq(\alpha(P), \omega(P))$ with $0 \in(\beta, \gamma)$. For each $s \in[\beta, \gamma]$ there exist $v_{s}>0, h_{s}>0$ such that the Taylor series expansion of $Z_{\mathbb{R}}(t, P)$ at $s$ converges absolutely for $t=t_{R}+i t_{I}$ with $\left|t_{R}-s\right|<h_{s}$ and $\left|t_{I}\right|<v_{s}$. Since $[\beta, \gamma]$ is covered by the open intervals $\left(s-h_{s}, s+h_{s}\right)$, there are $s_{j} \in[\beta, \gamma], j=1, \ldots, n$ such that $[\beta, \gamma] \subseteq \bigcup_{j=1}^{n}\left(s_{j}-h_{s_{j}}, s_{j}+h_{s_{j}}\right)$. Let $v=\min \left\{v_{s_{j}} \mid 1 \leq j \leq n\right\}$, so $\mathbb{Z}_{\mathbb{R}}(t, P)$ is analytic on $S:=\left\{t_{R}+i t_{I} \mid t_{R} \in\right.$ $\bigcup_{j=1}^{n}\left(s_{j}-h_{s_{j}}, s_{j}+h_{s_{j}}\right)$ and $\left.\left|t_{I}\right|<v\right\}$, an open rectangle in the complex plane containing $0 . S$ is star-shaped with respect to 0 and by uniqueness, $Z_{\mathbb{R}}(t, P)=Z_{\mathbb{C}}(t, P)$ on $S$, so $S \subseteq S_{P}$, i.e. $[\beta, \gamma] \subseteq S_{P}$.

Corollary 2.6. $Z_{\mathbb{C}}(t, P)=Z_{\mathbb{R}}(t, P)$ for $t \in(\alpha(P), \omega(P))$.

Hereafter, we will drop the subscripts and simply refer to $Z(t, P)$.

\section{Properties of $S_{P}$ And of $Z(t, P)$ on $S_{P}$}

Theorem 3.1. If $s \in \mathbb{C}-\{0\}$, then $s \cdot S_{s P}=S_{P}$ and

$$
Z(t, s P)=s Z(s t, P)
$$

for all $t \in S_{P}$.

Proof. By uniqueness we have for $t \in S_{s P} \cap s^{-1} S_{P}, Z(t, s P)=s Z(s t, P)$ since both sides satisfy $\frac{d Z}{d t}=Z^{2}$ and $Z(0)=s P$. Hence $Z(t, P)=Z\left(s s^{-1} t, P\right)=$ $s^{-1} Z\left(s^{-1} t, s P\right)$ if $t \in S_{P} \cap s S_{s P}$. Define $W(t, P)$ on $S_{P} \cup s S_{s P}$ by

$$
W(t, P):=\left\{\begin{array}{l}
Z(t, P) \text { if } t \in S_{P}, \\
s^{-1} Z\left(s^{-1} t, s P\right) \text { if } t \in s S_{s P} .
\end{array}\right.
$$

Then $W(t, P)$ is a solution to $(1.2)$ and $W(0, P)=P$. Hence $W(t, P) \equiv Z(t, P)$ and $S_{P} \cup s S_{s P}=S_{P}$ since $S_{P} \cup s S_{s P}$ is star-shaped with respect to 0 by Proposition 2.1(ii) and (iii). Hence $s S_{s P} \subseteq S_{P}$. Now replacing $P$ with $s^{-1} P$ gives $s S_{s\left(s^{-1} P\right)} \subseteq S_{s^{-1} P}$, i.e. $S_{P} \subseteq s^{-1} S_{s^{-1} P}$, and replacing $s^{-1}$ with $s$ gives $S_{P} \subseteq$ $s S_{s P}$.

Theorem 3.1 is the generalization of Theorem 1.8(ii) to the complex domain.

The next three results investigate the relationship between $S_{P}$ and $\left(\alpha\left(e^{i \theta} P\right)\right.$, $\left.\omega\left(e^{i \theta} P\right)\right)$ for $\theta \in[0,2 \pi]$. 
Corollary 3.2. $S_{P} \cap e^{i \theta} \cdot \mathbb{R}=e^{i \theta} \cdot\left(\alpha\left(e^{i \theta} P\right), \omega\left(e^{i \theta} P\right)\right)$ for $\theta \in[0,2 \pi]$.

Proof. By Theorem 3.1, $S_{P} \cap e^{i \theta} \cdot \mathbb{R}=e^{i \theta} \cdot S_{e^{i \theta} P} \cap e^{i \theta} \cdot \mathbb{R}=e^{i \theta}\left(S_{e^{i \theta} P} \cap \mathbb{R}\right)=$ $e^{i \theta} \cdot\left(\alpha\left(e^{i \theta} P\right), \omega\left(e^{i \theta} P\right)\right)$ by Theorem 2.5 .

Corollary 3.3. $\alpha\left(-e^{i \theta} P\right)=-\omega\left(e^{i \theta} P\right)$ and $\omega\left(-e^{i \theta} P\right)=-\alpha\left(e^{i \theta} P\right)$.

Proof.

$$
\begin{aligned}
e^{i \theta} \cdot\left(\alpha\left(e^{i \theta} P\right), \omega\left(e^{i \theta} P\right)\right) & =S_{P} \cap e^{i \theta} \mathbb{R}=S_{P} \cap e^{i(\theta-\pi)} \cdot \mathbb{R} \\
& =e^{i(\theta-\pi)}\left(\alpha\left(e^{i(\theta-\pi)} P\right), \omega\left(e^{i(\theta-\pi)} P\right)\right) \\
& =-e^{i \theta} \cdot\left(\alpha\left(-e^{i \theta} P\right), \omega\left(-e^{i \theta} P\right)\right) \\
& =e^{i \theta} \cdot\left(-\omega\left(-e^{i \theta} P\right),-\alpha\left(-e^{i \theta} P\right)\right) .
\end{aligned}
$$

\section{Corollary 3.4.}

$$
S_{P}=\bigcup_{\theta \in[0,2 \pi]} e^{i \theta}\left(\alpha\left(e^{i \theta} P\right), \omega\left(e^{i \theta} P\right)\right)=\bigcup_{\theta \in\left[-\frac{\pi}{2}, \frac{\pi}{2}\right]} e^{i \theta}\left(\alpha\left(e^{i \theta} P\right), \omega\left(e^{i \theta} P\right)\right) .
$$

The next theorem finishes the generalization of Theorem 1.8 to the complex domain.

Theorem 3.5. (i) If $A \in \operatorname{Aut}\left(\mathfrak{A}_{\mathbb{C}}\right)$, then $S_{P}=S_{A P}$ and for all $t \in S_{P}$,

$$
A Z(t, P)=Z(t, A P) \text {. }
$$

(ii) If $A \in A$ ut $\left(\mathfrak{A}_{\mathbb{C}}\right)$ and $P \in E_{1}(A)$, then $Z(t, P) \in E_{1}(A)$ for all $t \in S_{P}$.

(iii) Suppose $A \in \operatorname{Aut}\left(\mathfrak{A}_{\mathbb{C}}\right)$ and $P \in E_{a}(A)$. Then $S_{P}=a S_{P}$ and for all $t \in S_{P}$,

$$
A Z(t, P)=a Z(a t, P) .
$$

If a is not a root of 1 , then $S_{P}=\mathbb{C}$ and $Z(t, P)$ is a polynomial in $t$ (and $P$ ).

(iv) Suppose $D \in \operatorname{Der}\left(\mathfrak{A}_{\mathbb{C}}\right)$ and $P \in E_{a}(D)$ for some $a \neq 0$. Then $Z(t, P)$ is a polynomial in $t$ (and $P$ ) and $S_{P}=\mathbb{C}$.

Proof. (i) By uniqueness for $t \in S_{P} \cap S_{A P}, A Z(t, P)=Z(t, A P)$ since both sides satisfy (1.2) and the initial condition $Z(0)=A P$. Define $W(t, P)$ on $S_{P} \cup S_{A P}$ by

$$
W(t, P):=\left\{\begin{array}{l}
Z(t, P) \text { if } t \in S, \\
A^{-1} Z(t, A P) \text { if } t \in S_{A P} .
\end{array}\right.
$$

Then $W(t, P)$ is a solution to $(1.2)$ with $W(0, P)=P$. Thus $W(t, P) \equiv Z(t, P)$ and $S_{p} \cup S_{A P}=S_{P}$, so $S_{A P} \subseteq S_{P}$. Replacing $P$ with $A^{-1} P$ gives $S_{P} \subseteq S_{A^{-1} P}$ and replacing $A^{-1}$ with $A$ gives $S_{P} \subseteq S_{A P}$.

(ii): By (i), $A Z(t, P)=Z(t, A P)=Z(t, 1 \cdot P)=Z(t, P)$ for all $t \in S_{P}$.

(iii): $a S_{a P}=S_{P}=S_{A P}=S_{a P}$. The rest of the proof is the same as for Theorem 1.8; see [6] and [3].

\section{Complex conjugation}

Theorem 4.1. $\bar{S}_{P}=S_{\bar{P}}$ and for all $t \in S_{P}$,

$$
\overline{Z(t, P)}=Z(\bar{t}, \bar{P}) .
$$


Proof. If $t \in S_{P}$, then $t=s e^{i \theta}$ for some $\theta \in\left[-\frac{\pi}{2}, \frac{\pi}{2}\right]$ and $s \in\left(\alpha\left(e^{i \theta} P\right), \omega\left(e^{i \theta} P\right)\right)$, so $\bar{t}=s e^{-i \theta}$ and $s \in\left(\alpha\left(\overline{e^{i \theta} P}\right), \omega\left(\overline{e^{i \theta} P}\right)\right)=\left(\alpha\left(e^{-i \theta} \bar{P}\right), \omega\left(e^{-i \theta} \bar{P}\right)\right)$ by Corollary 1.9. Hence $\bar{t} \in S_{\bar{P}}$ by Corollary 3.4. Thus $\overline{S_{P}} \subseteq S_{\bar{P}}$, i.e. $S_{P} \subseteq \overline{S_{\bar{P}}}$. Replacing $P$ by $\bar{P}$ gives $S_{\bar{P}} \subseteq \overline{S_{\bar{P}}}=\overline{S_{P}}$, so $\overline{S_{\bar{P}}} \subseteq \overline{\overline{S_{P}}}=S_{P}$, so $S_{P}=\overline{S_{\bar{P}}}$.

Now if $t \in S_{P}$, then $t=s e^{i \theta}$ for some $\theta \in\left[-\frac{\pi}{2}, \frac{\pi}{2}\right]$ and $s \in\left(\alpha\left(e^{i \theta} P\right), \omega\left(e^{i \theta} P\right)\right)$. Then

$$
\begin{aligned}
\overline{Z(t, P)} & =\overline{Z\left(s e^{i \theta}, P\right)} \\
& =\overline{e^{-i \theta} Z\left(s, e^{i \theta} P\right)} \quad \text { by Theorem } 3.1 \\
& =\overline{e^{-i \theta}} \cdot Z\left(s, \overline{e^{i \theta} P}\right) \quad \text { by Corollary } 1.9 \\
& =e^{i \theta} \cdot Z\left(s, e^{-i \theta} \bar{P}\right) \\
& =e^{i \theta} \cdot e^{-i \theta} Z\left(s e^{-i \theta}, \bar{P}\right) \quad \text { by Theorem } 3.1 \\
& =Z(\bar{t}, \bar{P}) .
\end{aligned}
$$

We can see Theorem 4.1 very easily in the one-dimensional case.

Example 4.2. If $\operatorname{dim} \mathfrak{A}_{\mathbb{R}}=1$, then (1.2) is $\frac{d z}{d t}=z^{2}$ and the solution to (1.2) for which $z(0)=p$ is $z(t, p)=\frac{p}{1-t p}$ and $S_{p}=\mathbb{C}-\{t p \mid t \in[1, \infty)\}$. Hence $\overline{z(t, p)}=$ $\frac{\bar{p}}{1-t p}=\frac{\bar{p}}{1-\bar{t} \bar{p}}=z(\bar{t}, \bar{p})$ and $\bar{S}_{p}=\mathbb{C}-\{\overline{t p} \mid t \in[1, \infty)\}=\mathbb{C}-\left\{t \bar{p} \mid t \in[1, \infty]=S_{\bar{p}}\right.$.

Corollary 4.3. Suppose $P \in \mathfrak{A}_{\mathbb{R}}$. Then $S_{P}=\overline{S_{P}}$ and for all $t \in S_{P}$,

$$
\overline{Z(t, P)}=Z(\bar{t}, P) \text {. }
$$

Corollary 4.3 was also proven by Michael Kinyon (personal communication).

Corollary 4.4. Suppose $A \in A u t\left(\mathfrak{A}_{\mathbb{C}}\right)$ and for some $t_{0} \in S_{P}, \quad A Z\left(t_{0}, P\right)=$ $a Z\left(t_{0}, P\right)$. Then $\bar{A} Z\left(\bar{t}_{0}, \bar{P}\right)=\bar{a} Z\left(\bar{t}_{0}, \bar{P}\right)$. In particular, if $P \in \mathfrak{A}_{\mathbb{R}}$, then $Z\left(t_{0}, P\right) \in$ $E_{a}(A)$ iff $Z\left(\bar{t}_{0}, P\right) \in E_{\bar{a}}(\bar{A})$. If $P \in \mathfrak{A}_{\mathbb{R}}$ and $A \in \operatorname{Aut}\left(\mathfrak{A}_{\mathbb{R}}\right)$, then $Z\left(t_{0}, P\right) \in E_{a}(A)$ iff $Z\left(\bar{t}_{0}, P\right) \in E_{\bar{a}}(A)$.

Proof.

$$
\begin{aligned}
\bar{A} Z\left(\bar{t}_{0}, \bar{P}\right) & =\overline{A \overline{Z\left(\bar{t}_{0}, \bar{P}\right)}} \quad \text { by the definition of } \bar{A} \\
& =\overline{A Z\left(t_{0}, P\right)} \quad \text { by Theorem } 4.1 \\
& =\overline{a Z\left(t_{0}, P\right)} \\
& =\bar{a} Z\left(\bar{t}_{0}, \bar{P}\right) \quad \text { by Theorem } 4.1 .
\end{aligned}
$$

Example 4.5. It is easy to check that $F(X)=y^{3}-x^{3}$ for $X=\left(\begin{array}{l}x \\ y\end{array}\right)$ is a first integral for the differential equation (1.4.1); i.e. $F(X)$ is constant along the trajectories of (1.4.1). $A \in \operatorname{Aut}\left(\mathfrak{A}_{\mathbb{R}}\right)$, where $A\left(\begin{array}{l}x \\ y\end{array}\right):=\left(\begin{array}{l}y \\ x\end{array}\right)$ and $\mathfrak{A}_{\mathbb{R}}$ is $\mathbb{R}^{2}$ with multiplication defined by (1.4.2). Hence if $P \notin E_{1}(A)$, then the trajectory of $Z(t, P)=Z_{\mathbb{C}}(t, P)$ is the graph of $F(X)=F(P)$ since this curve has no equilibrium points on it. If $P \in \mathfrak{A}_{\mathbb{R}}, c=F(P)$, and $\alpha$ is a primitive cube root of 1 , then the trajectory of $Z(t, P)$ intersects $E_{-1}(A)$ at the three points $X_{1}, X_{2}, X_{3}$, where $X_{j}:=\alpha^{j}\left(\begin{array}{c}-\sqrt[3]{\frac{c}{2}} \\ \sqrt[3]{\frac{c}{2}}\end{array}\right)$ since $F\left(X_{j}\right)=c=F(P)$ for $j=1,2,3$. By Corollary 4.4 if $Z\left(t_{0}, P\right)=X_{1}$, then $Z\left(\bar{t}_{0}, P\right)=\bar{X}_{1}=X_{2}$. 
Example 4.5 also shows that Corollary 3.6 of [3], which states that the trajectory of $Z(t, P)$ intersects $E_{a}(A)$ for $a \neq 1, A \in \operatorname{Aut}\left(\mathfrak{A}_{\mathbb{R}}\right)$, in at most two points, is no longer true once we allow $t$ to take on complex values. Thus some of the results of [3] do not generalize to the complex case.

\section{Trouble in Paradise: Shifts}

The following result follows from the fact that (1.2) is autonomous.

Proposition 5.1. Suppose $s \in(\alpha(P), \omega(P))$ and $Z(s, P)=Q$. Then $(\alpha(Q), \omega(Q))$ $=(\alpha(P)-s, \omega(P)-s)$ and

$$
Z(t+s, P)=Z(t, Z(s, P))
$$

for all $t \in(\alpha(P)-s, \omega(P)-s)$.

The problem in the complex case is that $S_{P}-s$ may not be star-shaped with respect to 0 . We can see this in the one-dimensional case.

Example 5.2. Returning to Example 4.2 we have $z(t, 1)=\frac{1}{1-t}$ and $S_{1}=\mathbb{C}-\{t \mid t \in$ $[1, \infty)\}$. Thus $i \in S_{1}$, but $S_{1}-i=\mathbb{C}-\{t-i \mid t \in[1, \infty)\}$ is not star-shaped with respect to 0 . Moreover $z(i, 1)=\frac{1}{1-i}=\frac{1}{2}(1+i)$ and $S_{\frac{1}{2}(1+i)}=\mathbb{C}-\left\{\frac{1}{2} t(1+i) \mid t \in\right.$ $[1, \infty)\}$.

However, it is possible to generalize Proposition 5.1 to a certain extent.

Theorem 5.3. Suppose $T \subseteq S_{P}, s \in T$, and $T$ is open. If $T$ is star-shaped with respect to $s$, then $T-s \subseteq S_{Q}$, where $Q=Z(s, P)$ and $T-s:=\{t-s \mid t \in T\}$. Moreover, for $t \in T-s$,

$$
Z(t+s, P)=Z(t, Z(s, P)) .
$$

Proof. Since $T$ is star-shaped with respect to $s, T-s$ is star-shaped with respect to 0 , and $t \in T-s$ iff $t+s \in T$. Define $W(t, Q)$ on $T-s$ by $W(t, Q):=Z(t+s, P)$. Then $W(t, Q)$ satisfies $(1.2)$ and $W(0, Q)=Q$, so $W(t, Q)=Z(t, Q)=Z(t, Z(s, P))$ and $T-s \subseteq S_{Q}$.

Corollary 5.4. (i) Suppose $R$ is the radius of convergence of the Maclauren series of $Z(t, P)$. Let $B_{R}:=\{t \in \mathbb{C}|| t \mid<R\}$. If $|s|<R$ and $Q=Z(s, P)$, then $B_{R}-s \subseteq S_{Q}$.

(ii) If $S_{P}=\mathbb{C}$ and $Q=Z(s, P)$ for some $s \in \mathbb{C}$, then $S_{Q}=\mathbb{C}$.

Corollary 5.5. Suppose $s \in S_{P}$ and $Z(s, P)=Q$. Then $-s \in S_{Q}$ and $Z(-s, Q)=$ $P$.

Proof. By an argument similar to that in the proof of Theorem 2.5, there is an open rectangle $T \subseteq S_{P}$ with $0, s \in T$. Then by Theorem 5.3, $T-s \subseteq S_{Q}$. Since $-s \in T-s$, by (5.3.1), $Z(-s, Q)=Z(-s, Z(s, P))=Z(-s+s, P)=Z(0, P)=$ $P$.

Corollary 5.6. Suppose there is an $A \in A$ ut $\left(\mathfrak{A}_{\mathbb{C}}\right)$ such that $A P=Z(s, P)$ for some $s \in S_{P}$. Suppose $T \subseteq S_{P}, T$ is open and $T$ is star-shaped with respect to $s$. Then $T-s \subseteq S_{P}$. In particular $-s \in S_{P}$.

Proof. This follows from Theorem 5.3, Corollary 5.5 and Theorem 3.5(i), where $Q=A P$. 
Corollary 5.7. Suppose there is an $A \in A$ ut $\left(\mathfrak{A}_{\mathbb{C}}\right)$ such that $A P=Z(s, P)$ for some $s \in S_{P}$ with $s \neq 0$. If $s=r e^{i \theta}$, where $r \in \mathbb{R}$, then $\left(\alpha\left(e^{i \theta} P\right), \omega\left(e^{i \theta} P\right)\right)=\mathbb{R}$.

Proof. $A e^{i \theta} P=e^{i \theta} Z(s, P)=Z\left(r, e^{i \theta} P\right)$ by Theorem 3.1. Then

$$
\begin{aligned}
\left(\alpha\left(e^{i \theta} P\right), \omega\left(e^{i \theta} P\right)\right) & =\left(\alpha\left(A e^{i \theta} P\right), \omega\left(A e^{i \theta} P\right)\right) \text { by Theorem 1.8(iii) } \\
& =(\alpha(Q), \omega(Q)) \text { where } Q=Z\left(r, e^{i \theta} P\right) \\
& =\left(\alpha\left(e^{i \theta} P\right)-r, \omega\left(e^{i \theta} P\right)-r\right) \text { by Proposition 5.1. }
\end{aligned}
$$

Hence $\alpha\left(e^{i \theta} P\right)=-\infty$ if $r>0$ and $\omega\left(e^{i \theta} P\right)=\infty$ if $r<0$.

If $r<0$, then $A^{n} e^{i \theta} P=Z\left(n r, e^{i \theta} P\right)$ for $n \in \mathbb{N}$ by induction using (5.1.1), so $n s \in S_{P}$ for $n \in \mathbb{N}$ and $A^{n} P=Z(n s, P)$ and hence $-n s \in S_{P}$ by Corollary 5.6. Hence $\alpha\left(e^{i \theta} P\right)=-\infty$ and $\left(\alpha\left(e^{i \theta} P\right), \omega\left(e^{i \theta} P\right)\right)=\mathbb{R}$ if $r<0$.

If $r>0$ we have $A^{-1} P=Z(-s, P)$ and the result follows from the argument in the previous paragraph using $A^{-1}$ in place of $A$. That $A^{-1} P=Z(-s, P)$ follows from $A Z(-s, P)=Z(-s, A P)=Z(-s, Z(s, P))=Z(-s+s, P)=Z(0, P)=P$ using (5.1.1).

It is easy to get an example in which the hypotheses of Corollary 5.6 are true.

Example 5.8. By Proposition 5.3 of $[6]$ if $D \in \operatorname{Der}\left(\mathfrak{A}_{\mathbb{R}}\right)$ such that $D P=P^{2}$, then $(\alpha(P), \omega(P))=\mathbb{R}$ and $Z(t, P)=e^{t D} P$. Similarly, if $D \in \operatorname{Der}\left(\mathfrak{A}_{\mathbb{C}}\right)$ such that $D P=P^{2}$, then $S_{P}=\mathbb{C}$ and $Z(t, P)=e^{t D} P$. In this case if $A=e^{s D}$, then $A P=e^{s D} P=Z(s, P)$.

Example 5.9. Suppose $\mathfrak{A}_{\mathbb{R}}=M_{n}(\mathbb{R}), n \times n$ matrices over $\mathbb{R}$ and the right-hand side of (1.2) is the matrix product square of $Z$. In this case we denote the product on $M_{n}(\mathbb{R})$ coming from (1.3) with o; thus

$$
Z \circ W=\frac{1}{2}(Z W+W Z) .
$$

It is well known 12 that $Z(t, P)=(\text { id }-t L(P))^{-1} P$, where $\operatorname{id}(P)=P$ and $L(P) X=P \circ X$. Now if $B \in G L_{n}(\mathbb{C})$, then $A \in$ Aut $\left(\mathfrak{A}_{\mathbb{C}}\right)$, where $A(Z):=B Z B^{-1}$. Then $A P=Z(s, P)$ iff $B P B^{-1} \circ(I-s P)=P$ for some $s \in S_{P}$.

\section{SOME PERIODICITY RESUlts}

Theorem 6.1. Suppose $S_{P}=\mathbb{C}$. Then $Z(t, P)$ is periodic iff there are $t_{0}, t_{1} \in \mathbb{C}$ with $t_{0} \neq t_{1}$ and $Z\left(t_{0}, P\right)=Z\left(t_{1}, P\right)$.

Proof. If $Z(t, P)$ is periodic, then $Z(0, P)=Z(T, P)$, where $T \neq 0$ is the period. Conversely, if $Z\left(t_{0}, P\right)=Z\left(t_{1}, P\right)$ for some $t_{0} \neq t_{1}$, then by Theorem 5.3 and Corollary 5.4(ii), $Z(t, P)=Z\left(t-t_{0}+t_{0}, P\right)=Z\left(t-t_{0}, Z\left(t_{0}, P\right)\right)=Z\left(t-t_{0}, Z\left(t_{1}, P\right)\right)=$ $Z\left(t-t_{0}+t_{1}, P\right)$ for all $t \in \mathbb{C}$, so $Z(t, P)$ is periodic.

Theorem 6.2. Suppose $S_{P}=\mathbb{C}$ and there is an $A \in A$ ut $\left(\mathfrak{A}_{\mathbb{C}}\right)$ such that $A P=$ $Z\left(t_{0}, P\right)$ for some $t_{0} \neq 0$. If $|A|<\infty$, then $Z(t, P)$ is periodic.

Proof. For all $t \in \mathbb{C}$,

$$
\begin{aligned}
A Z(t, P) & =Z(t, A P) \quad \text { by Theorem } 3.5(\mathrm{i}) \\
& =Z\left(t, Z\left(t_{0}, P\right)\right) \\
& =Z\left(t+t_{0}, P\right) \quad \text { by Theorem 5.3. }
\end{aligned}
$$


By induction $A^{j} Z(t, P)=Z\left(t+j t_{0}, P\right)$ for $j \in \mathbb{N}$. Hence if $A^{n}=\mathrm{id}, Z(t, P)=$ $Z\left(t+n t_{0}, P\right)$, so $Z(t, P)$ is periodic.

Lemma 6.3. Suppose $S_{P}=\mathbb{C}$ and there is an $A \in \operatorname{Aut}\left(\mathfrak{A}_{\mathbb{C}}\right)$ such that $A Z\left(t_{0}, P\right)=$ $a Z\left(t_{0}, P\right)$ for some $t_{0} \in \mathbb{C}$ and $a \neq 1$. Then for all $t \in \mathbb{C}$,

$$
A Z(t, P)=a Z\left(a t+(1-a) t_{0}, P\right) .
$$

Proof. This follows from Theorems 5.3, 3.5(i), and 3.1.

Theorem 6.4. Suppose $P \in \mathfrak{A}_{\mathbb{R}}, S_{P}=\mathbb{C}$, and $A \in A$ ut $\left(\mathfrak{A}_{\mathbb{R}}\right)$ such that $A Z\left(t_{0}, P\right)=$ $-Z\left(t_{0}, P\right)$ for some $t_{0} \notin \mathbb{R}$. Then $Z(t, P)$ is periodic.

Proof. We have $A Z(t, P)=-Z\left(-t+2 t_{0}, P\right)$ by Lemma 6.3 . Since $\bar{P}=P$ and $\bar{A}=A$, we have $A Z\left(\bar{t}_{0}, P\right)=-Z\left(t_{0}, P\right)$ by Corollary 4.4, so again using Lemma 6.3 gives $A Z(t, P)=-Z\left(-t+2 \bar{t}_{0}, P\right)$ for all $t \in \mathbb{C}$. Thus $Z\left(2 t_{0}, P\right)=Z\left(2 \bar{t}_{0}, P\right)$, so $Z(t, P)$ is periodic by Theorem 6.1 since $2 t_{0} \neq 2 \bar{t}_{0}$.

Note that the period in Theorem 6.4 is purely imaginary.

Example 6.5. Continuing with Examples 1.4 and 4.5, if $P \in \mathfrak{A}_{\mathbb{R}}$ and $P \notin E_{1}(A)$, then either $S_{P} \neq \mathbb{C}$ or $Z(t, P)$ is periodic.

Theorem 6.6. (i) Suppose $D \in \operatorname{Der}\left(\mathfrak{A}_{\mathbb{C}}\right), P \in \mathfrak{A}_{\mathbb{R}}$ and $P \in E_{a}(D)$ for $a \neq 0$. If $P^{2} \neq 0, A \in A$ ut $\left(\mathfrak{A}_{\mathbb{R}}\right)$ and $t_{0} \notin \mathbb{R}$, then $Z\left(t_{0}, P\right) \notin E_{-1}(A)$.

(ii) Suppose $B \in \operatorname{Aut}\left(\mathfrak{A}_{\mathbb{C}}\right), P \in \mathfrak{A}_{\mathbb{R}}$ and $P \in E_{b}(B)$ for $b$ not a root of 1 . If $A \in \operatorname{Aut}\left(\mathfrak{A}_{\mathbb{R}}\right), t_{0} \notin \mathbb{R}$, and $P^{2} \neq 0$, then $Z\left(t_{0}, P\right) \notin E_{-1}(A)$.

Proof. For both (i) and (ii), Theorem 3.5 implies that $Z(t, P)$ is a nonconstant polynomial in $t$, so the result follows from Theorem 6.4.

Finally we have the following result.

Proposition 6.7. Suppose $P \in \mathfrak{A}_{\mathbb{R}}, S_{P}=\mathbb{C}$ and suppose $Z\left(t_{0}, P\right) \in \mathfrak{A}_{\mathbb{R}}$ for some $t_{0} \notin \mathbb{R}$. Then $Z(t, P)$ is periodic.

Proof.

$$
\begin{aligned}
\overline{Z\left(t_{0}, P\right)} & =Z\left(\bar{t}_{0}, P\right) \quad \text { by Corollary } 4.3 \\
& =Z\left(t_{0}, P\right) \quad \text { since } Z\left(t_{0}, P\right) \in \mathfrak{A}_{\mathbb{R}}
\end{aligned}
$$

and the result follows from Theorem 6.1 since $t_{0} \neq \bar{t}_{0}$.

\section{ACKNOWLEDGEMENT}

The author would like to thank Michael Kinyon for much help on this topic and also Elizabeth Brown for her help with $\S 2$. The author also thanks the Mathematics Department at Indiana University Bloomington for their continued research support.

\section{REFERENCES}

[1] E. Hille, Ordinary differential equations in the complex domain, Dover Publications, Inc., Mineola, NY, 1997. Reprint of the 1976 original. MR1452105 (97m:34001)

[2] N. C. Hopkins, Quadratic differential equations in the complex domain II, forthcoming.

[3] N. C. Hopkins and M. K. Kinyon, Automorphism eigenspaces of quadratic differential equations and qualitative theory, Differential Equations Dynam. Systems 5 (1997), no. 2, 121-138. MR 1657250 (99i:34017) 
[4] E. L. Ince, Ordinary Differential Equations, Dover Publications, New York, 1944. MR.0010757 $(6,65 f)$

[5] N. Jacobson, Forms of algebras, Some Recent Advances in the Basic Sciences, Vol. 1 (Proc. Annual Sci. Conf., Belfer Grad. School Sai., Yeshiva Univ., New York, Unknown Month 1962), Belfer Graduate School of Science, Yeshiva Univ., New York, 1966, pp. 41-71. MR0214628 (35 \#5477)

[6] M. K. Kinyon and A. A. Sagle, Quadratic dynamical systems and algebras, J. Differential Equations 117 (1995), no. 1, 67-126, DOI 10.1006/jdeq.1995.1049. MR.1320184 (96e:34018)

[7] M. K. Kinyon and A. A. Sagle, Automorphisms and derivations of differential equations and algebras, Rocky Mountain J. Math. 24 (1994), no. 1, 135-154, DOI 10.1216/rmjm/1181072457. 20th Midwest ODE Meeting (Iowa City, IA, 1991). MR.1270032 (95d:34015)

[8] M. K. Kinyon and A. A. Sagle, Differential systems and algebras, Differential equations, dynamical systems, and control science, Lecture Notes in Pure and Appl. Math., vol. 152, Dekker, New York, 1994, pp. 115-141. MR1243197(94i:34025)

[9] M. K. Kinyon and S. Walcher, On ordinary differential equations admitting a finite linear group of symmetries, J. Math. Anal. Appl. 216 (1997), no. 1, 180-196, DOI 10.1006/jmaa.1997.5668. MR.1487259 (99c:34011)

[10] L. Marcus, Quadratic differential equations and nonassociative algebras, in Contributions to the Theory of Nonlinear Oscillations, Vol. V, L. Cesair, J.P. LaSalle, and S. Lefschetz (eds.), Princeton Univ. Press, Princeton, 1960, 185-213.

[11] R. D. Schafer, An introduction to nonassociative algebras, Pure and Applied Mathematics, Vol. 22, Academic Press, New York-London, 1966. MR0210757 (35 \#1643)

[12] S. Walcher, Algebras and differential equations, Hadronic Press Monographs in Mathematics, Hadronic Press, Inc., Palm Harbor, FL, 1991. MR1143536 (93e:34002)

Department of Mathematics and Computer Science, Indiana State University, Terre HAUTE, INDIANA 47809 